\title{
COVID-19 und die Langzeitfolgen
}

Lunge, Herz, Niere, zentrales Nervensystem - das SARS-CoV-2 infiziert als „Multiorganvirus“ die unterschiedlichsten Organe. Dementsprechend sind viele Langzeitfolgen denkbar. Vor allem bei schweren Verläufen sollten die Patienten daher intensiv nachbetreut werden.

Nicht einmal ein halbes Jahr nach dem bisherigen Höhepunkt der Coronavirus-Pandemie in Deutschland sind die Spätfolgen von COVID-19 Mitte Juli 2020 noch schwer abzuschätzen. Es zeichnet sich aber ab, dass sich einige Patienten über Monate nicht vollständig regenerieren - und dass unter innen auch Menschen mit milden COVID-19-Verläufen sind. "Genesen“ bedeutet im Fall von COVID-19 also nicht immer "geheilt".

) Es zeichnet sich ab, dass sich einige Patienten über Monate nicht vollständig regenerieren

Der Virologe Professor Peter Piot, Direktor der London School of Hygiene and Tropical Medicine, infizierte sich im März mit SARS-CoV-2. Dem Fachmagazin "Science" [1] berichtete er von noch wochenlang persistierenden Symptomen: „Nach einem schweren COVID-19-Verlauf entwickelte ich eine Lungenerkrankung, verursacht durch den durch das Virus ausgelösten

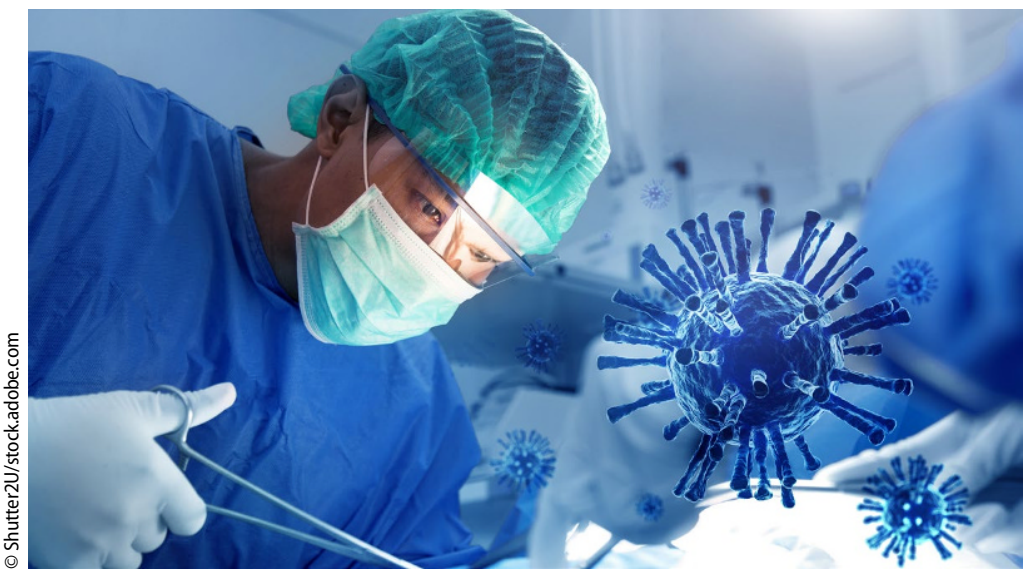

Abb. $1 \Delta$ Da SARS-CoV-2 nicht nur die Lunge infiziert und zu einer systemischen Endotheliitis führen kann, sind Langzeitfolgen in mehreren Organsystemen möglich. (Symbolbild mit Fotomodell)

Zytokin-Sturm. Ich werde immer noch mit hohen Dosen von Kortikosteroiden behandelt. Ich leide unter Vorhofflimmern, das behandelt werden muss, auch um Thrombosen und einen Schlaganfall zu verhindern."

\section{"So einfach ist es nicht"}

Piot, der sich mehr als die Hälfte seines Lebens mit Viren beschäftigt hat, warnte in dem Bericht eindringlich: „Viele Menschen glauben, dass ein Prozent der Infizierten stirbt und der Rest mit milden, Grippe-ähnlichen Symptomen davonkommt. Aber so einfach ist es nicht: Viele Patienten werden chronische Nieren- und Herzerkrankungen entwickeln, weltweit wird es Tausende geben, die für den Rest ihres Lebens behandelt werden müssen."

Da das Virus nicht nur die Lunge infiziert, sondern auch andere Organsysteme wie Herz, Niere oder das ZNS und durch eine überschießende Immunantwort zu einer systemischen Endotheliitis führen kann, sind Lang-

Quelle: Anne Bäurle Ärzte Zeitung zeitfolgen in mehreren Organsystemen möglich (• Abb. 1), etwa:

\section{Pulmonale Folgeerkrankungen}

SARS-CoV-2 verursacht ja sehr häufig Atemwegsinfektionen. Einige $\mathrm{Pa}$ tienten berichten auch bei einem milden Verlauf noch über einige Wochen über Kurzatmigkeit, und gerade bei Patienten, die beatmet werden müssen, können längerfristige Schäden durch die oft über mehrere Wochen notwendige Beatmung auftreten.

\) "Genesen" bedeutet im Fall von COVID-19 nicht immer "geheilt"

Auch kommt es bei einer mechanischen Beatmung häufiger zu Koinfektionen - auch diese können bekanntlich mögliche Langzeitfolgen haben. Mehrere Studien, die unter anderem auch in Deutschland durchgeführt werden, untersuchen derzeit die Spätfolgen auf die Lunge.

\section{Neurologische Schäden}

Auch bei milden Verläufen kann SARSCoV-2 neurologische Schäden verursachen, allerdings wohl eher selten. So hat eine aktuelle Studie ergeben, dass eine COVID-19-Erkrankung die neuroaxonale Integrität auch bei Erwachsenen mit mildem bis moderaten Verlauf beeinträchtigen kann.

Bekannt ist auch, dass unter COVID-19 das Schlaganfallrisiko nicht nur deutlich erhöht ist, sondern dass die Hirninsulte auch gravierender als üblich sind [2]. Dadurch können auch motorische sowie Sprach- und Sehstörungen zurückbleiben.

Die auch bei milden Verläufen recht häufigen Geruchs- und Geschmacks- 
störungen persistieren in vielen Fällen noch über Wochen, wie eine Studie mit 200 Patienten ergeben hat: Auch fünf bis sechs Wochen nach Beginn eine COVID-19-Erkrankung hatten über ein Drittel noch anhaltende Riech- und Schmeckdefizite.

Jeder Zehnte mit solchen Beeinträchtigungen spürte dabei überhaupt keine Verbesserung [3]. Inwieweit der Geruchs- und Geschmacksverlust tatsächlich eine Langzeitfolge ist, muss allerdings noch beobachtet werden auch bei einer Influenza kann dieses Symptom persistieren.

\section{Nierenschäden}

Besonders bei schwerkranken beatmungspflichtigen COVID-19-Patienten wird das Auftreten von akutem und zum Teil dialysepflichtigen Nierenversagen beobachtet. Virologe Piot sagte gegenüber dem Magazin Science, er rechne mit tausenden $\mathrm{Pa}$ tienten, die in Folge der Pandemie lebenslänglich auf eine Dialyse angewiesen sein könnten.

Ein Problem könnte zudem sein, dass gerade junge Patienten nach ihrer Genesung die Nierenbeeinträchtigungen zunächst nicht bemerken und erst mit zunehmendem Alter die Folgen erkennbar werden.

\section{Herz-Kreislauf-Erkrankungen}

Eine kardiale Beteiligung lässt sich anhand erhöhter Herzenzyme beziehungsweise Troponin bei einem Teil der Patienten nachweisen, darunter auch Kinder und Patienten mit mildem oder moderatem Verlauf, berichtet das RKI.

Besonders bei schweren Infektionen der Atemwege erleide eine Reihe von Patienten kardiovaskuläre Erkrankungen, einschließlich Myokardschädigungen, Myokarditis, akutem Myokardinfarkt, Herzinsuffizienz, Herzrhythmusstörungen und venösen thromboembolischen Ereignissen.

Die pathologisch erhöhte Blutgerinnung geht bei schweren COVID19-Verläufen mit einem erhöhten
Risiko für Thromboembolien, sowie Lungenarterien- und zerebrovaskulären Embolien und möglichen Folgeschäden einher.

Noch ist nicht abzusehen, wie häufig Folgeschäden tatsächlich auftreten, dafür gibt es die COVID-19-Erkrankung noch nicht lang genug. Gerade Patienten mit schweren Verläufen das sind nach bisherigen Schätzungen etwa $14 \%$ aller COVID-19-Patienten sollten allerdings intensiv nachbetreut werden.

) Noch ist nicht abzusehen, wie häufig Folgeschäden tatsächlich auftreten

Da allerdings auch bei leichten Symptomen Folgeerkrankungen auftreten können, gilt sowohl für Ärzte als auch Patienten: Auch nach der Genesung auf Symptome achten!

\section{Literatur}

1. Draulans D: Finally, a virus got me. Scientist who fought Ebola and HIV reflects on facing death from COVID-19. Sciencemag.org. https://doi.org/10.1126/science.abc7042

2. Ntaios $G$, Michel P, Georgiopoulos $G$ (2020) Characteristics and Outcomes in Patients With COVID-19 and Acute Ischemic Stroke. https://doi.org/10.1161/STROKEAHA. 120.031208

3. Boscolo-Rizzo P, Borsetto D, Fabbris C, et al (2020) Evolution of Altered Sense of Smell or Taste in Patients With Mildly Symptomatic COVID-19. JAMA Otolaryngol Head Neck Surg. https://doi.org/10.1001/jamaoto.2020.1379

\section{Hier steht eine Anzeige.}

Hinweis des Verlags. Der Verlag bleibt in Hinblick auf geografische Zuordnungen und Gebietsbezeichnungen in veröffentlichten Karten und Institutsadressen neutral.

rheuma plus $2020 \cdot 19: 162-163$ https://doi.org/10.1007/s12688-02000369-9

○) Springer-Verlag GmbH Austria, ein Teil von Springer Nature 2020 